\title{
Stabilization of a Model Formalinized Protein Antigen Encapsulated in Poly(lactide-co-glycolide)-Based Microspheres
}

\author{
WENLEI JIANG, ${ }^{1,2}$ STEVEN P. SCHWENDEMAN ${ }^{1}$ \\ ${ }^{1}$ Department of Pharmaceutical Sciences, The University of Michigan, Ann Arbor, Michigan 48109-1065 \\ ${ }^{2}$ Division of Pharmaceutics, College of Pharmacy, The Ohio State University, Columbus, Ohio 43210
}

Received 10 November 2000; accepted 5 March 2001

\begin{abstract}
A formaldehyde-mediated aggregation pathway (FMAP) has been shown to be primarily responsible for the solid-state aggregation of lyophilized formalinized protein antigens [e.g., tetanus toxoid (TT) and formalinized bovine serum albumin (f-BSA)] in the presence of moisture and physiological temperature. Coincorporation of the formaldehyde-interacting amino acid, histidine, strongly inhibits the FMAP. The purpose of this study was to test whether previous solid-state data are applicable toward the stabilization of formalinized antigens encapsulated in poly(lactide-co-glycolide) (PLGA)-based microspheres. Formaldehyde-treated bovine serum albumin (f-BSA) and BSA were selected as a model formalinized protein antigen and a nonformalinized control, respectively. As in the solid state, we found that the FMAP was dominant in the aggregation of f-BSA encapsulated in PLGA 50/50 microspheres, whereas the aggregation mechanism of encapsulated BSA was mostly converted from thiol-disulfide interchange to an acid-catalyzed noncovalent pathway. The lack of noncovalent aggregation in encapsulated f-BSA could be explained by its higher thermodynamic stability after formalinization, which inhibits protein unfolding. Targeting the FMAP, coencapsulation of histidine and trehalose successfully inhibited the aggregation of f-BSA in microspheres. By combining the use of an optimized oil-in-oil (o/o) encapsulation method, coencapsulation of histidine and trehalose, and use of low-acid-content poly(D,L-lactide) (PLA) and poly(ethylene glycol) (PEG) blends, a 2-month continuous release of fBSA was achieved with the absence of aggregation. (c) 2001 Wiley-Liss, Inc. and the American Pharmaceutical Association J Pharm Sci 90:1558-1569, 2001

Keywords: formaldehyde-mediated aggregation pathway (FMAP); microspheres; poly(lactideco-glycolide); poly(D,L-lactide); poly(ethylene glycol); formalinized antigens
\end{abstract}

\section{INTRODUCTION}

Poly(lactide-co-glycolide) (PLGA) microspheres have shown great potential as carriers for singledose vaccine delivery and the advantages of PLGA microspheres are well known. ${ }^{1-3}$ A major obstacle in developing this delivery system is the instability of encapsulated protein antigens during

Correspondence to: S.P. Schwendeman (Telephone: 734615-6574; Fax: 734-615-6162; E-mail: schwende@umich.edu)

Journal of Pharmaceutical Sciences, Vol. 90, 1558-1569 (2001)

() 2001 Wiley-Liss, Inc. and the American Pharmaceutical Association exposure to physiological conditions (i.e., elevated humidity and temperature). Protein antigens may undergo hydrolysis, oxidation, deamidation, and aggregation in polymer devices, resulting in the loss of antigenicity or incomplete release of active antigens. ${ }^{1,4}$ For example, in the development of controlled-release tetanus toxoid (TT) microspheres, the incomplete release and antigenicity losses of TT were attributed to the aggregation and acid-induced denaturation of the antigen inside microspheres. ${ }^{5,6}$

The stability of protein antigens in PLGA systems is rather complicated. It is not only related to the physical and chemical properties 
of antigens but also affected by the physical and chemical processes during microsphere preparation, storage, and in vivo antigen release. ${ }^{1}$ In addition to characteristics determined by their original primary and higher order structures, some protein antigens may acquire additional properties from their preparation procedures, which may cause some additional instability problems. For example, formaldehyde treatment has been commonly used to convert toxins into relatively inert but still antigenic toxoids. Formaldehyde-treated antigens, [e.g., TT, formalinized bovine serum albumin (f-BSA), and formalinized ribonuclease A (f-RNase)] have been shown to possess special reactive species that trigger a unique formaldehyde-mediated aggregation at intermediate moisture levels. ${ }^{7,8}$ Briefly, labile antigen-formaldehyde linkages formed during formaldehyde treatment are converted to highly reactive electrophiles (e.g., Schiff bases) under appropriate conditions. When lyophilized antigens are incubated at elevated humidity and temperature, Schiff bases may combine with nucleophiles within the same or different protein molecules to form intra- and intermolecular crosslinks, eventually leading to the formation of nondisulfide-linked covalently bonded insoluble aggregates. We term this unique process the formaldehyde-mediated aggregation pathway (FMAP). Our previous work demonstrated coincorporation of strongly formaldehyde-interacting amino acids (e.g., histidine) as well as prior reduction of f-BSA with cyanoborohydride can significantly inhibit the FMAP. ${ }^{8}$

Formalinized protein antigens represent a large family of vaccine antigens. The formalinized antigens, TT and diphtheria toxoid (DT), are two top priority antigens of the World Health Organization for the development of single-dose vaccine formulations. ${ }^{9}$ For more than a decade, research efforts have intensified toward the formulation design of a controlled-release TT vaccine. However, no ideal continuous release of antigenic TT has been achieved, largely because of the antigen instability in polymeric systems. Many excipients aimed at improving the microsphere microclimate (e.g., BSA, Poloxamer 188, and basic salts) have been coencapsulated with TT to improve antigen stability. ${ }^{10,11}$ Various kinds of stabilizers (e.g., heparin, dextran, and trehalose) have also been tested for their capacity to maintain the antigenicity of TT after lyophilization. ${ }^{12}$ However, few studies have considered the influence of formalinization on the stability of TT in PLGA systems. ${ }^{13}$ It is unclear if formaldehyde-mediated aggregation processes will occur after antigens are encapsulated in PLGA microspheres. Since the FMAP is general to any formaldehyde-treated protein antigen, the answer to this question may be beneficial to such antigens undergoing single-dose vaccine development.

The objective of this study was to investigate the stability of encapsulated formalinized antigens and to answer the question whether the FMAP occurs in PLGA microspheres. Moreover, if the FMAP is dominant, corresponding stabilization strategies will be developed against the FMAP in PLGA microspheres. The final goal of this study is to achieve continuous release of formalinized antigen from microspheres with the absence of aggregation. As in our previous study, f-BSA was used as a model antigen to be encapsulated in PLGA microspheres for the stability investigation. To delineate formaldehyde-mediated aggregation, BSA was employed as a nonformalinized control. ${ }^{8}$

\section{EXPERIMENTAL SECTION}

\section{Materials}

Poly(D,L-lactide-co-glycolide) (PLGA) 50/50 with inherent viscosity (iv) of 0.20 and $0.64 \mathrm{dL} / \mathrm{g}$ in hexafluoroisopropanol and poly(D,L-lactide) (PLA) with iv of $1.07 \mathrm{dL} / \mathrm{g}$ in $\mathrm{CHCl}_{3}$ were from BPI (Birmingham, AL). Bovine serum albumin (BSA; A-3059, Lot 32H0463) was purchased from Sigma Chemical Company (St. Louis, MO). Formalinized bovine serum albumin (f-BSA) was developed in our lab by treating BSA with formaldehyde for 3 weeks. ${ }^{8}$ All other biochemicals and chemicals were of analytical grade or purer and obtained from commercial suppliers.

\section{Microencapsulation}

The polymeric microspheres were prepared by either a water-in-oil-in-water double emulsionsolvent evaporation (w/o/w) method or an oil-in-oil single emulsion-solvent extraction (o/o) method. In the w/o/w method, an aqueous solution of antigen in $1 \mathrm{mM}$ pH 7.3 phosphate buffer was emulsified into a methylene chloride-PLGA 50/50 solution by homogenization. Then $1 \%$ poly(vinyl alcohol) (PVA) aqueous solution was added to the first w/o emulsion and the second w/o/w emulsion was formed by vortex at high speed. 
This emulsion was poured into a hardening aqueous bath containing $0.3 \%$ PVA and stirred for $3 \mathrm{~h}$ at room temperature and atmospheric pressure. The hardened microspheres were collected by centrifugation, washed with double distilled water, and lyophilized over 2 days. In the anhydrous o/o method, dried protein powders were directly suspended in acetonitrile-polymer solution. The lyophilization protocol to prepare the dried protein powder was as follows: protein aqueous solutions in $1 \mathrm{mM} \mathrm{pH} 7.3$ phosphate buffer were flash frozen in liquid $\mathrm{N}_{2}$ and placed on a Labconco Freeze Dryer (Kansas City, MO) operating at $30 \mu \mathrm{m}$ of $\mathrm{Hg}$ pressure and a condenser temperature of $-46^{\circ} \mathrm{C}$. Typically, protein samples were removed after $48 \mathrm{~h}$. Then, a uniform suspension of sieved antigen powder $(<45 \mu \mathrm{m})$ in acetonitrile-polymer solution was homogenized at 15,000 rpm in an ice bath. The antigen suspension was added in a dropwise manner into the continuous phase [cottonseed oil containing $1.6 \%(\mathrm{w} / \mathrm{v})$ span 85] stirred at $750 \mathrm{rpm}$ with an overhead stirrer. After $5 \mathrm{~h}$, petroleum ether (bp, $50-110^{\circ} \mathrm{C}$ ) was poured into the cottonseed oil bath to extract the acetonitrile from the polymer. After an additional 15 min of stirring, the microspheres were filtered, washed with $250 \mathrm{~mL}$ of petroleum ether and lyophilized.

\section{Scanning Electron Microscopy (SEM) of Microspheres}

The microspheres were coated with gold-palladium by using Pelco model 3 sputter coater 91000 . Surface morphology of the microspheres was examined by a Philips XL Scanning Electron Microscope.

\section{Determination of Antigen Loading in Microspheres}

The amount of antigen encapsulated in microspheres was determined by recovering the protein directly from the microspheres. First, acetone was added in microspheres to dissolve the polymer. The mixture was vortexed and centrifuged and then supernatant was removed. After the removal of polymer was repeated three times, the remaining protein pellet was reconstituted in PBS containing $0.02 \%$ Tween $80^{\circledR}$ (PBST) and protein content was determined by the Coomassie Plus method (Pierce Chem Co., Rockford, IL). Encapsulation efficiency for the w/o/w method was between 49 and $90 \%$ and for the o/o method between 90 and $100 \%$.

\section{Evaluation of Model Antigen Aggregation in Microspheres Exposed to a Humid Environment}

Ten milligrams of microspheres were incubated at $37^{\circ} \mathrm{C}$ and 80 or $97 \%$ relative humidity $(\mathrm{RH})$. Saturated $\left(\mathrm{NH}_{4}\right)_{2} \mathrm{SO}_{4}$ and $\mathrm{K}_{2} \mathrm{SO}_{4}$ aqueous solutions placed in glass dessicators were used to attain 80 and $97 \% \mathrm{RH}$, respectively. ${ }^{14}$ Microspheres were collected periodically, and the soluble protein content in microspheres was determined as described in Determination of Antigen Loading in Microspheres. Any insoluble protein aggregates were collected by centrifugation and reconstituted in denaturing agent $[8 \mathrm{M}$ urea or $6 \mathrm{M}$ guanidine. $\mathrm{HCl}(\mathrm{GnCl})]$. Determination of any aggregates soluble in denaturing agent gave the amount of noncovalently bonded aggregates. With the further addition of reducing agent (10 mM DTT + $1 \mathrm{mM}$ EDTA), any disulfidebonded aggregates were dissolved. The total dissolved portion in denaturing and reducing agents gave the total amount of noncovalent and disulfide-bonded aggregates. Any remaining aggregates undissolved in combined denaturing and reducing agents were considered as formaldehyde-mediated aggregates. ${ }^{8}$

\section{Evaluation of Antigen Release From Microspheres}

Samples of microspheres (20 mg) were suspended in $1 \mathrm{~mL}$ of PBST. The suspension was incubated at $37^{\circ} \mathrm{C}$ with mild agitation. At preselected intervals, release media were removed for protein assay and replaced with fresh buffer. The amount of protein released was assayed by the Coomassie Plus method. At the end of release, the protein remaining in the polymer was determined as discussed in the next section.

\section{Evaluation of Antigen Aggregation in Microspheres During Release}

Microspheres were incubated in PBST at $37^{\circ} \mathrm{C}$. At predetermined time intervals, the release medium was removed for determination of protein content, and microspheres were collected and dried. The remaining protein in the polymer was analyzed as described in Evaluation of Model Antigen Aggregation in Microspheres Exposed to a Humid Environment. 


\section{Chemical Denaturation of Model Antigens by $\mathrm{GnCl}^{15}$}

For $\mathrm{GnCl}$ unfolding of BSA and f-BSA, $100 \mu \mathrm{g} / \mathrm{mL}$ of protein stock solution and $8 \mathrm{M} \mathrm{GnCl}$ were prepared in $30 \mathrm{mM}$ MOPS buffer (pH 7.0). Protein stock solution was diluted in various concentrations of $\mathrm{GnCl}$ to reach the final protein concentration of $6.25 \mu \mathrm{g} / \mathrm{mL}$. The fluorescence of model antigens at $345 \mathrm{~nm}$ was determined when the protein was excited at $280 \mathrm{~nm}$ by using a Fluromax-2 flurometer (Instrument S. A., Inc., Edison, $\mathrm{NJ}$ ). The $C_{\mathrm{m}}$ parameter, the $\mathrm{GnCl}$ concentration at which the unfolded fraction of protein is 0.5 , was determined from the unfolding curve (unfolding fraction plotted against $\mathrm{GnCl}$ concentration). Unfolding fraction can be calculated by the following equation:

$$
\alpha=\frac{F_{\mathrm{N}}-F_{i}}{F_{\mathrm{N}}-F_{\mathrm{U}}}
$$

where $\alpha$ is the unfolded fraction of the protein; $F_{\mathrm{N}}$ and $F_{\mathrm{U}}$ are the fluorescence of the native (in absence of $\mathrm{GnCl}$ ) and fully unfolded states (in concentrated $\mathrm{GnCl}$ ), respectively; and $F_{i}$ is the protein fluorescence at the $i$ th concentration of $\mathrm{GnCl}$.

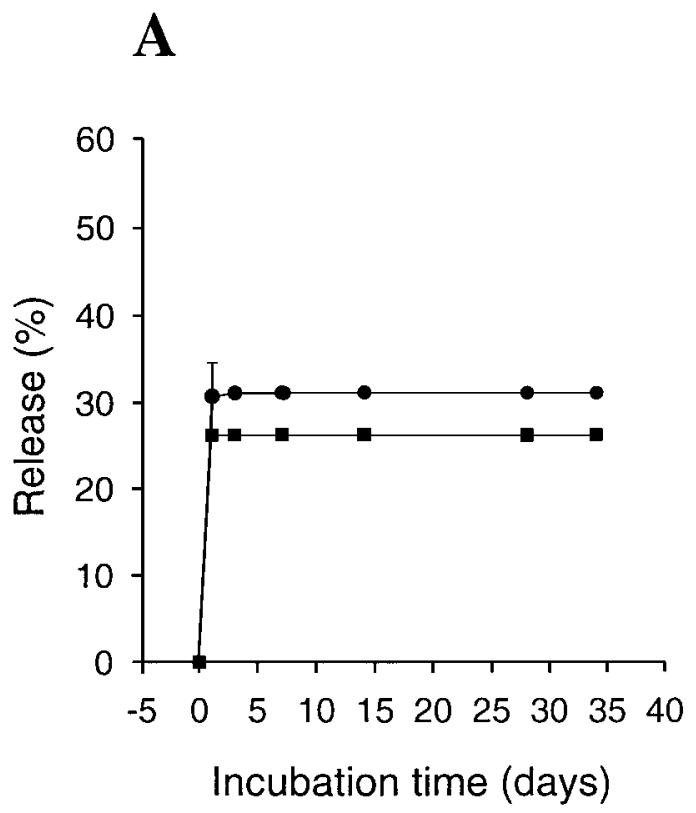

Figure 1. (A) Cumulative release kinetics of BSA ( and f-BSA ( $\mathbf{a}$ ) from $1.5 \%$ protein/PLGA50/50 (iv $=0.20$ $\mathrm{dL} / \mathrm{g}$ ) w/o/w microspheres at $37^{\circ} \mathrm{C}$ (average $\pm \mathrm{SD}, n=3$ ). (B) Aggregation of BSA (open bar) and f-BSA (solid

\section{RESULTS AND DISCUSSION}

\section{Aggregation Kinetics of Model Antigens in PLGA}

To test the stability of encapsulated f-BSA, we first encapsulated the antigen in PLGA 50/50 (iv $=0.20 \mathrm{dL} / \mathrm{g}$ ) by the most commonly used w/o/w method. To distinguish between the FMAP and typical aggregation pathways, we also encapsulated BSA as a control. Protein loading in both formulations was $1.5 \%$.

BSA and f-BSA microspheres were incubated in PBST at $37^{\circ} \mathrm{C}$. The release of protein antigens and the loss of soluble antigens due to aggregation in the microspheres were monitored simultaneously. As seen in Figure 1A, incomplete release of BSA and f-BSA ( $\sim 30 \%$ of antigens) was observed. In the polymer, a slow growth of insoluble aggregates of both BSA and f-BSA appeared (Figure 1B). By 28 days, $\sim 40 \%$ of initially encapsulated BSA had become insoluble and unreleasable from the polymer. Insoluble f-BSA inside microspheres increased from 8 to $31 \%$ after 7 and 28 days of incubation.

To evaluate the stability of encapsulated antigens in the moist polymer, microspheres were incubated at $80 \% \mathrm{RH}$ and $37^{\circ} \mathrm{C}$. Protein release

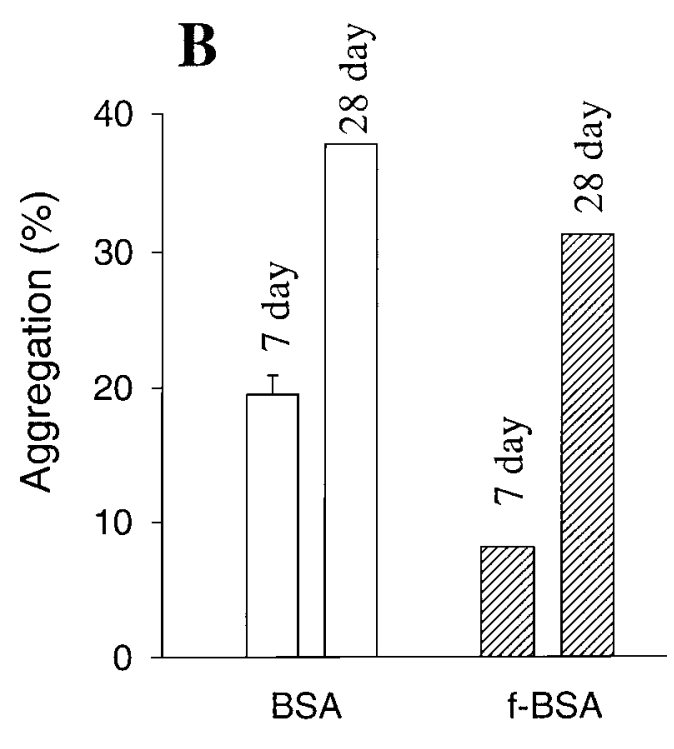

bar) ${ }^{27}$ in $1.5 \%$ protein/PLGA50/50 (iv $=0.20 \mathrm{dL} / \mathrm{g}$ ) w/o $/ \mathrm{w}$ microspheres when incubated at $37^{\circ} \mathrm{C}$ and in the release medium (average $\pm \mathrm{SD}, n=3$ ). 
was presumably prevented under these conditions. As seen in Figure 2, a growing amount of insoluble aggregates were observed in both BSA and f-BSA microspheres. However, f-BSA had much faster aggregation kinetics. By 7 days, only $6.9 \%$ of f-BSA remained soluble in microspheres, whereas BSA lost $\sim 80 \%$ of soluble protein in microspheres after 28 days of incubation.

Therefore, each antigen, BSA and f-BSA, slowly formed aggregates inside microspheres both during release and when incubated at elevated humidity.

\section{Aggregation of $\mathrm{f}$-BSA Encapsulated in PLGA via the FMAP}

The aggregates formed in PLGA microspheres during the previous deleterious conditions (release and humidity) were solublized in denaturing and reducing solvents to elucidate the aggregation pathway for each antigen (Table 1). As reported in the solid state, ${ }^{8}$ virtually none $(<11 \%)$ of the aggregates formed from encapsulated f-BSA in PLGA microspheres were soluble in combined denaturing and reducing agents $(8 \mathrm{M}$ urea + $10 \mathrm{mM}$ DTT + $1 \mathrm{mM}$ EDTA), after f-BSAcontaining microspheres were incubated in either the release medium or the humid condition $(80 \%$ $\mathrm{RH})$ for 28 days. Therefore, this insolubility of $\mathrm{f}$ BSA aggregates demonstrates that the FMAP does occur for f-BSA in microspheres.

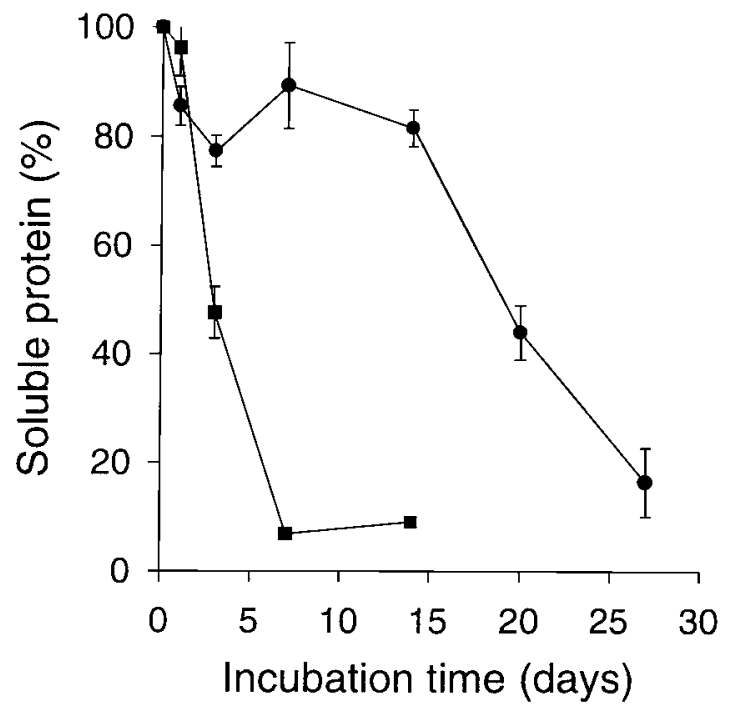

Figure 2. Aggregation kinetics of BSA (-) and fBSA $(\square)^{27}$ in $1.5 \%$ protein/PLGA50/50 (iv $\left.=0.20 \mathrm{dL} / \mathrm{g}\right)$ w/o/w microspheres after exposure to $80 \% \mathrm{RH}$ and $37^{\circ} \mathrm{C}$. (average $\pm \mathrm{SD}, n=3$ ).
In contrast, aggregates formed from the encapsulated control antigen, BSA, were always soluble in the combined reducing and denaturing solvents irrespective of condition used, although the distribution of noncovalent to disulfide-bonded aggregation for BSA was altered depending on the deleterious conditions (Table 1). For example, after 28 days of release, $76 \%$ of BSA aggregates formed in the polymer were soluble in denaturing agents ( $8 \mathrm{M}$ urea), indicating that the noncovalent aggregates had formed, whereas remaining aggregates were completely soluble in the combined denaturing and reducing agents $(8 \mathrm{M}$ urea $+10 \mathrm{mM}$ DTT $+1 \mathrm{mM}$ EDTA), indicating the minor involvement of thiol-disulfide interchange. For BSA microspheres incubated at $37^{\circ} \mathrm{C}$ and $80 \% \mathrm{RH}$, the same type of water-insoluble aggregates were observed among the remaining BSA (Table 1), except a higher number of disulfide-bonded aggregates were observed. Thus, examination of the aggregation behavior of the nonformalinized antigen confirms that f-BSA underwent a different aggregation pathway from BSA in PLGA microspheres.

Comparing the stability of encapsulated antigens during release with that under humid conditions, aggregation was more extensive in the latter case. This increased aggregation was possibly because the antigen release from microspheres was inhibited during humidity exposure, thereby increasing the residence time of antigens in the polymer and the probability of aggregation. When incubated at elevated humidity and $37^{\circ} \mathrm{C}$, as in the release medium, PLGA degradation still occurs, because of uptake of water vapor at $37^{\circ} \mathrm{C}$, and a similar deleterious microenvironment will be produced, although at a different rate. In both cases, encapsulated protein antigens will be continuously exposed to elevated moisture levels, polymer hydrophobic surfaces, and an acidic $\mathrm{pH}$ because of the accumulation of acidic polymer degradation products. ${ }^{17}$ Our data suggest that the difference in incubation conditions was not great enough to alter the aggregation mechanisms corresponding to either of the model antigens. Therefore, the humidity condition can be a relatively simpler but useful model system to study the antigen aggregation mechanism, as shown previously. ${ }^{18}$ In addition, by using humidity studies, stabilization strategies may be analyzed mechanistically and stabilizers can be identified more efficiently. Antigen stability data obtained from humidity experiments will also provide important 
Table 1. Solubility of Aggregates of Antigens in Denaturing and Reducing Solvents

\begin{tabular}{|c|c|c|c|c|}
\hline Antigen Formulations & $\begin{array}{l}\text { Experimental } \\
\text { Conditions }\end{array}$ & $\begin{array}{l}\text { Reconstituting } \\
\text { Solvent }\end{array}$ & $\begin{array}{l}\text { Aggregate Solubility } \\
(\%)(\text { Ave } \pm \mathrm{SD}, n=3)^{b}\end{array}$ & $\begin{array}{l}\text { Aggregation } \\
\text { Mechanism }\end{array}$ \\
\hline f-BSA & Release medium, & $8 \mathrm{M}$ urea & $11 \pm 4$ & FMAP \\
\hline$(1.5 \%$ f-BSA/w/o/w) & $37^{\circ} \mathrm{C}, 28$ days & $8 \mathrm{M}$ urea+10 mM DTT & $11 \pm 4$ & \\
\hline f-BSA & $80 \% \mathrm{RH}$ & $8 \mathrm{M}$ urea & $3.5 \pm 0.2$ & FMAP \\
\hline$(1.5 \% \mathrm{f}-\mathrm{BSA} / \mathrm{w} / \mathrm{o} / \mathrm{w})$ & $37^{\circ} \mathrm{C}, 28$ days & $8 \mathrm{M}$ urea+10 mM DTT & $3.1 \pm 0.6$ & \\
\hline $\mathrm{f}-\mathrm{BSA}^{a}$ & $80 \% \mathrm{RH}$ & $8 \mathrm{M}$ urea & 0 & FMAP \\
\hline No polymer & $37^{\circ} \mathrm{C}, 12$ days & $8 \mathrm{M}$ urea+10 mM DTT & $8 \pm 2$ & \\
\hline BSA & Release medium, & $8 \mathrm{M}$ urea & $76 \pm 4$ & Non-covalent, \\
\hline$(1.5 \% \mathrm{BSA} / \mathrm{w} / \mathrm{o} / \mathrm{w})$ & $37^{\circ} \mathrm{C}, 28$ days & $8 \mathrm{M}$ urea+10 mM DTT & $99 \pm 4$ & Disulfide bonded \\
\hline BSA & $80 \% \mathrm{RH}$ & $8 \mathrm{M}$ urea & $46 \pm 5$ & Non-covalent, \\
\hline$(1.5 \% \mathrm{BSA} / \mathrm{w} / \mathrm{o} / \mathrm{w})$ & $37^{\circ} \mathrm{C}, 28$ days & $8 \mathrm{M}$ urea+10 mM DTT & $100 \pm 20$ & Disulfide bonded \\
\hline $\mathrm{BSA}^{a}$ & $80 \% \mathrm{RH}$ & $8 \mathrm{M}$ urea & 0 & \\
\hline No polymer & $37^{\circ} \mathrm{C}, 12$ days & $8 \mathrm{M}$ urea+10 mM DTT & $92 \pm 6$ & Disulfide bonded \\
\hline
\end{tabular}

${ }^{a}$ Data reported previously. ${ }^{8}$

${ }^{b}$ The percentage of aggregates solubility was calculated from the amount of soluble protein recovered in different solvents relative to the total amount of protein aggregates.

information with respect to microsphere storage conditions.

\section{Comparison of Protein Aggregation in the Nonencapsulated Solid-State and in PLGA}

When compared with aggregation of nonencapsulated antigens in the solid state, the aggregation mechanism of encapsulated BSA and f-BSA showed different changes. As seen in Table 1, nonencapsulated BSA formed aggregates almost exclusively via the thiol-disulfide interchange aggregation pathway, evidenced by $92 \%$ aggregates soluble in combined denaturing and reducing agents, but no aggregates soluble in denaturing agent alone. In contrast, encapsulated BSA aggregates were mostly noncovalent and partially disulfide-bonded. For f-BSA, the FMAP dominated in both nonencapsulated and encapsulated f-BSA samples.

Different from its nonencapsulated counterparts, encapsulated protein antigens were continuously exposed to the deleterious microsphere microclimate. How does the deleterious microclimate suppress the thiol-disulfide interchange initiated by a single free thiol group in BSA, but promote the noncovalent interaction between BSA molecules? Previous work in our lab has demonstrated that it is the acidic microclimate and intermediate moisture levels in microspheres that cause noncovalent aggregation of BSA. ${ }^{18,19}$ BSA is known to undergo a conformational transition from the $\mathrm{F}$ (fast) to $\mathrm{E}$ (expanded) isoforms at $\mathrm{pH}$ 2.7. In the $\mathrm{E}$ form, more hydrophobic area is exposed, which provides the driving force for noncovalent aggregation via hydrophobic interactions. In addition to the conformational change, the nucleophilic free thiolate becomes protonated due to the acidic $\mathrm{pH}$ in microspheres, contributing to the conversion of the BSA aggregation mechanism.

f-BSA has a secondary and tertiary structure similar to that of BSA. ${ }^{8}$ Why did the acidic polymer microclimate not change the f-BSA aggregation behavior significantly? From the kinetic point of view, formaldehyde-mediated aggregation is so fast ( $>90 \%$ of f-BSA became insoluble within 1 week) that the acidic microclimate may have had insufficient time to build up. Thus, a reduced effect of the acidic microclimate on the aggregation of $\mathrm{f}-\mathrm{BSA}$ is expected. In addition, after formaldehyde treatment, formaldehyde linkages within f-BSA molecule are expected to rigidify its structure; thus, f-BSA may be conformationally more stable and less unlikely to unfold at the acidic microclimate. We confirmed this hypothesis by performing a $\mathrm{GnCl}$ denaturation curve on BSA and f-BSA. As seen in Figure 3, f-BSA has a much higher $C_{\mathrm{m}}$ (the $\mathrm{GnCl}$ concentration at which the unfolded fraction of protein is 0.5) than BSA, indicating that f-BSA has a more stable conformation than BSA. Evidently, intraprotein formaldehyde linkages do retard the conformational transitions of f-BSA, which is consistent with the report by Paliwal et al. that the conformation of the 


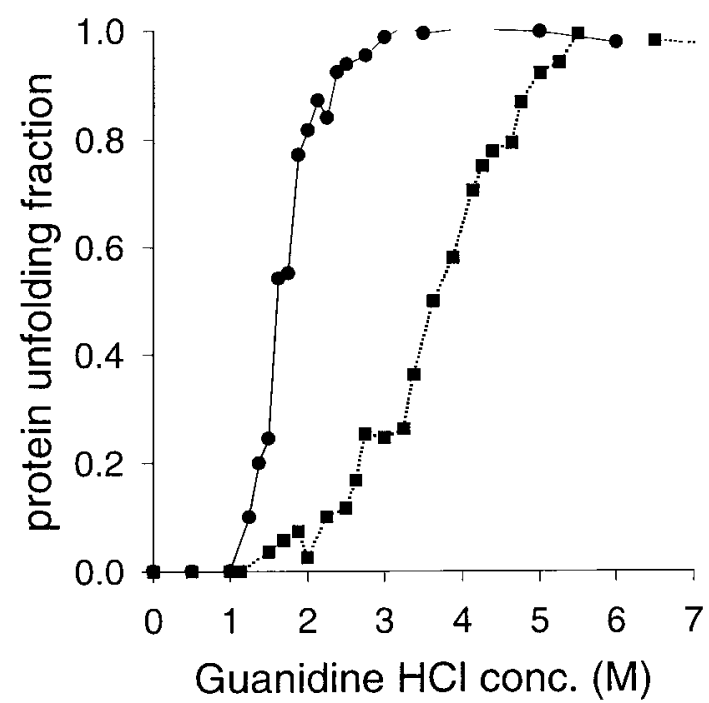

Figure 3. GnCl-induced unfolding transitions of BSA ( ) and f-BSA (ם) measured by fluorescence spectroscopy.

diphtheria toxoid was stabilized by formalinization and did not undergo a conformational change as easily as diphtheria toxin at acidic $\mathrm{pH}^{20}$

\section{Stabilization of f-BSA Encapsulated in the Polymer}

The challenge to formulate stable vaccine antigens in PLGA systems has stimulated considerable interest in the biotechnology and formulation fields. Our stabilization approach is to understand the mechanisms of antigen instability in PLGA systems first, instead of or in combination with, traditional trial-and-error methodologies. For f-BSA, we understand that formald- ehydemediated reactivity does initiate aggregation in PLGA (Figures 1 and 2; Table 1). Combining general instability sources pertaining to PLGA systems with specific instability factors for f-BSA, the most important instability sources for encapsulated f-BSA are enumerated as follows: (1) physical and chemical properties intrinsic to formalinized antigens (i.e., formaldehydemediated reactivity); (2) the encapsulation process; and (3) the microsphere environment. As we mentioned before, the deleterious microclimate did not change the aggregation behavior of f-BSA significantly. Thus, in the sections to follow we focused on stabilization strategies targeting the first two aspects.

\section{Enhanced Stability of f-BSA Afforded by the O/O Encapsulation Method}

Among the microencapsulation techniques, the $\mathrm{w} / \mathrm{o} / \mathrm{w}$ method is one of the most convenient ways to encapsulate proteins in PLGA microspheres. However, the presence of the aqueous phase may increase the diffusion of protein to the external phase, resulting in the decreased encapsulation efficiency, especially when high protein loading is required. Furthermore, proteins in general become more labile when confronting the organic solvent-water interface. ${ }^{21}$ Compared with the w/o/w method, negligible water content in the o/o system may significantly increase the encapsulation efficiency and protein stability. With less flexibility for unfolding, aggregation, and chemical reactions in the solid state, proteins display enhanced stability when dispersed in pure organic solvents. ${ }^{22,23}$ Thus, we also developed an o/o method to encapsulate f-BSA and compared the stability of f-BSA in o/o microspheres with those prepared by the w/o/w method.

To achieve higher loading, PLGA 50/50 (iv $=0.64 \mathrm{dL} / \mathrm{g}$ ) was selected to encapsulate model antigens and the theoretical loading of f-BSA was $8 \%$ in both $\mathrm{o} / \mathrm{o}$ and $\mathrm{w} / \mathrm{o} / \mathrm{w}$ encapsulation methods. In the o/o method, the polymer concentration in acetonitrile was $10 \%$, and $98 \pm 4 \%(n=3)$ encapsulation efficiency was achieved. In the w/o/w method, polymer concentration in methylene chloride was $30 \%$ and encapsulation efficiency was only $49 \pm 3 \%(n=3)$. In Table 2 , the influence of encapsulation methods on f-BSA stability at $97 \% \mathrm{RH}$ and $37^{\circ} \mathrm{C}$ is shown. In the w/o/w formulation, after 16 days of incubation, only $51 \pm 2 \%$ of f-BSA remained soluble in microspheres, whereas $112 \pm 1 \%$ of $\mathrm{f}$-BSA remained soluble in microspheres. Excellent stability of f-BSA o/o microspheres suggests that the o/o method is superior to the $\mathrm{w} / \mathrm{o} / \mathrm{w}$ method for antigen stability.

\section{Inhibition of f-BSA Aggregation by Histidine and Trehalose}

The second strategy applied targeted the specific formaldehyde-mediated reactivity intrinsic to formalinized antigens. It has been shown that formaldehyde-mediated aggregation can be inhibited by (1) blocking nucleophiles and/or reducing electrophiles in protein molecules by chemical modification; ${ }^{7,8}$ (2) adding low MW nucleophiles (amino acids) to compete for the reactive electrophiles in protein molecules; ${ }^{8}$ and (3) adding sugar 
Table 2. Effect of Encapsulation Method, Histidine, and Trehalose on f-BSA Aggregation in Microspheres After Exposure to $80 \% \mathrm{RH}$ and $37^{\circ} \mathrm{C}$

\begin{tabular}{|c|c|c|}
\hline Antigen Formulations & $\begin{array}{l}\text { Incubation } \\
\text { Condition }\end{array}$ & $\begin{array}{c}\text { Soluble f-BSA } \\
(\%)^{d}\end{array}$ \\
\hline \multicolumn{3}{|l|}{ Effect of encapsulation method } \\
\hline f-BSA w/o/w microspheres ${ }^{a}$ & $97 \% \mathrm{RH}, 37^{\circ} \mathrm{C}, 16$ days & $50 \pm 2$ \\
\hline f-BSA o/o microspheres ${ }^{a}$ & $97 \% \mathrm{RH}, 37^{\circ} \mathrm{C}, 16$ days & $112 \pm 1$ \\
\hline \multicolumn{3}{|l|}{ Effect of histidine and trehalose } \\
\hline f-BSA o/o microspheres ${ }^{a}$ & $80 \% \mathrm{RH}, 37^{\circ} \mathrm{C}, 6$ days & $30 \pm 5$ \\
\hline \multirow[t]{2}{*}{$\mathrm{f}-\mathrm{BSA}+$ His o/o microspheres ${ }^{a, b}$} & $80 \% \mathrm{RH}, 37^{\circ} \mathrm{C}, 6$ days & $106 \pm 10$ \\
\hline & $80 \% \mathrm{RH}, 37^{\circ} \mathrm{C}, 28$ days & $81 \pm 5$ \\
\hline \multirow[t]{2}{*}{ f-BSA+His+Tre o/o microspheres ${ }^{a, c}$} & $80 \% \mathrm{RH}, 37^{\circ} \mathrm{C}, 6$ days & $127 \pm 7$ \\
\hline & $80 \% \mathrm{RH}, 37^{\circ} \mathrm{C}, 28$ days & $102 \pm 14$ \\
\hline
\end{tabular}

${ }^{a}$ Theoretical protein loading was $8 \%$; PLGA50/50 (iv $\left.=0.64 \mathrm{dL} / \mathrm{g}\right)$ was used for microsphere preparation.

${ }^{6}$ Histidine was co-encapsulated in microspheres. The weight ratio of histidine to f-BSA was 1:5.

${ }^{c}$ Histidine and trehalose were co-encapsulated in microspheres. The histidine:trehalose:f-BSA weight ratio was 0.5:0.5:5.0.

${ }^{d}$ Soluble in PBST; data represent average $\pm \mathrm{SD}, n=3$.

molecules that may inhibit aggregation by several mechanisms. ${ }^{24}$ Sorbitol has been shown previously to inhibit the aggregation of lyophilized TT effectively. ${ }^{7,24}$ In addition, histidine was found as the most efficient nucleophilic stabilizer (it completely inhibits the aggregation of nonencapsulated f-BSA after 35 days of incubation at $80 \% \mathrm{RH}$ and $\left.37^{\circ} \mathrm{C}\right){ }^{8}$ In addition to its high nucleophilicity at neutral $\mathrm{pH}$, the ability to strongly interact with slowly released formaldehyde and form a stable reaction product allows histidine to inhibit the FMAP most effectively. Compared with chemical modification, the second and third strategies are more feasible because the chemically modified formalinized antigens may have altered antigenicity. Therefore, in this study, we decided to coencapsulate histidine and a sugar additive with f-BSA in microspheres and investigate if the inhibition of FMAP will be successful in the polymer device.

Before coencapsulating excipients in microspheres, we needed to identify a sugar that can significantly inhibit the aggregation of f-BSA. A series of sugars, including sucrose, trehalose, sorbitol, mannitol, and dextrose, was tested for their impact on the stability of lyophilized f-BSA. These sugars were co-lyophilized with f-BSA separately as a weight ratio of $1: 5$ and then protein powders were incubated at $37^{\circ} \mathrm{C}$ and $80 \%$ $\mathrm{RH}$. After 14 days of incubation, it was shown that coincorporation of either sorbitol or trehalose was most effective, largely increasing the soluble
f-BSA percentage from 9\% (without additive) to $30 \%$ (with additive) (data not shown). In addition, trehalose has been extensively reported to stabilize protein antigens during both lyophilization and microencapsulation processes. The possible mechanisms include stabilizing dried protein structure by replacing $\mathrm{H}$-bonding sites on the protein previously occupied by water, increasing the glass transition temperature of the formulation, and promoting preferential hydration of the antigen. ${ }^{25}$ Thus, trehalose was considered for coincorporation with histidine in the f-BSA-containing microspheres.

Before microsphere preparation, f-BSA was colyophilized with histidine only (the weight ratio of f-BSA to histidine was 5:1) or with both histidine and trehalose (the f-BSA/histidine/trehalose weight ratio was 5:0.5:0.5). Microspheres with $8 \%$ f-BSA loading were prepared by the o/o method. In Table 2, the loss of soluble protein in microspheres with time of incubation at $37^{\circ} \mathrm{C}$ and $80 \% \mathrm{RH}$ is displayed. Without any stabilizers, $\mathrm{f}$ BSA lost $70 \%$ of its solubility after 6 days of incubation, whereas f-BSA coencapsulated with histidine and f-BSA coencapsulated with histidine and trehalose still remained 81 and $102 \%$ soluble after 28 days of incubation, respectively. These data strongly suggested that histidine successfully inhibited the FMAP in PLGA systems and the addition of trehalose improved the effectiveness of histidine to stabilize f-BSA in PLGA systems. 

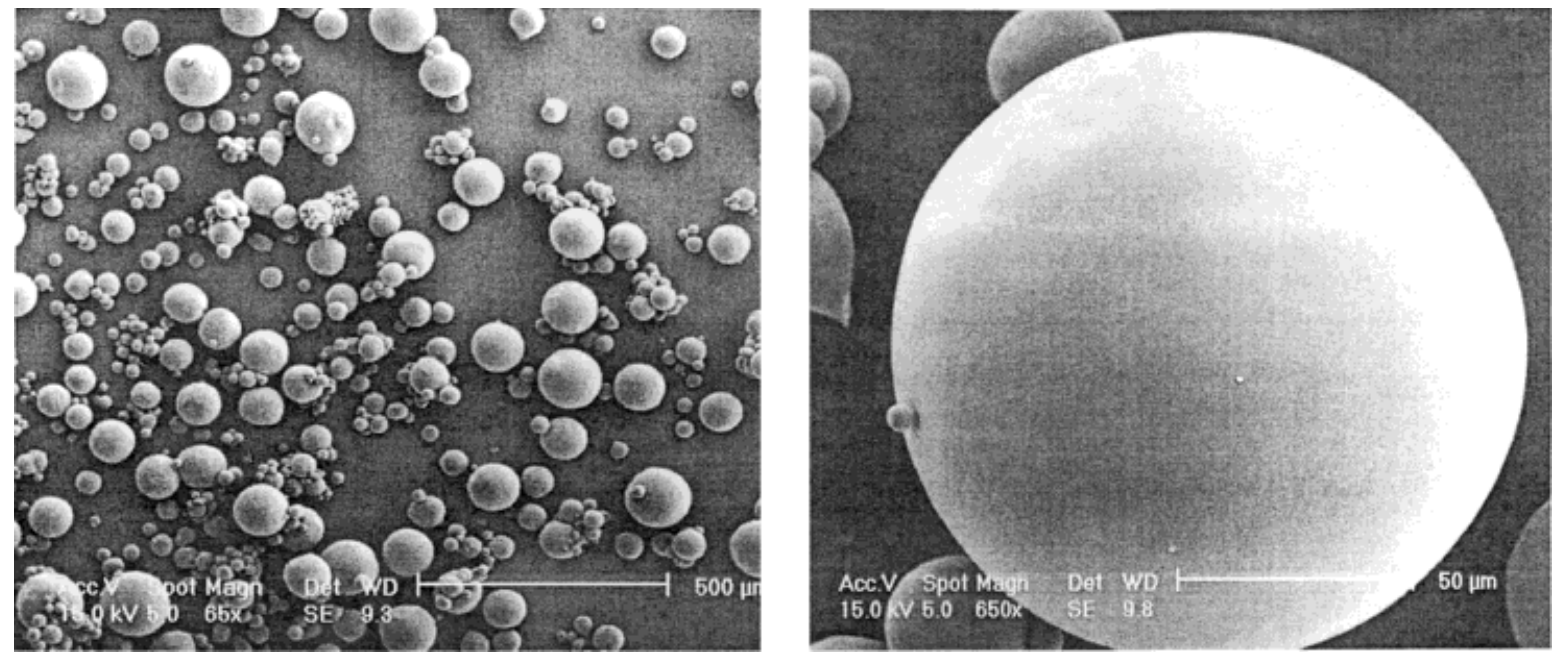

Figure 4. SEM images of $6.1 \% \mathrm{f}-\mathrm{BSA}+$ histidine + trehalose/PLA $(\mathrm{iv}=1.07 \mathrm{dL} / \mathrm{g}) /$ PEG35,000 o/o microspheres.

\section{Controlled Release of f-BSA From Low-Acid-Content Microspheres Without Aggregation}

After identifying the specific formulation [8\% f-BSA/histidine/trehalose/PLGA 50/50 (iv $=0.64$ $\mathrm{dL} / \mathrm{g}$ o/o microspheres) that stabilized f-BSA at intermediate moisture levels, we aimed at obtaining continuous release of stable f-BSA from microspheres. The release kinetics of this stabilized f-BSA formulation was tested. On the first day, $80 \%$ of f-BSA was released and the large burst effect could not be reduced even when the loading was decreased to 5\% (data not shown). An additional problem for this formulation was the expected low $\mathrm{pH}$ microclimate in PLGA 50/50 microspheres during release. ${ }^{19}$ Although acidic $\mathrm{pH}$ does not aggravate the FMAP, ${ }^{8}$ it may induce peptide-bond hydrolysis and other degradation actions. In addition, most bacterial toxoids are generally unstable below $\mathrm{pH} 4.5,{ }^{6}$ making it desirable for protein antigens to be released from a neutral microenvironment. Therefore, the final goal of this study was to devise a microsphere formulation which could (1) inhibit the aggregation of f-BSA, (2) release f-BSA continuously, and (3) strongly suppress the acidic microenvironment during f-BSA release.

We have previously reported the use of a PLA/ PEG blend o/o formulation which (1) successfully eliminates the acid-induced noncovalent aggregation and peptide hydrolysis of BSA, and (2) provides a slow and continuous release of BSA for 4 weeks. ${ }^{26}$ This formulation is suggested to have a more neutral microclimate than typical PLGA 50/50 microspheres because acid-labile BSA is stable in the PLA/PEG blend system. In addition, strong water uptake of PEG significantly increases the water content in the blend. Therefore, the PLA/PEG blend formulation with

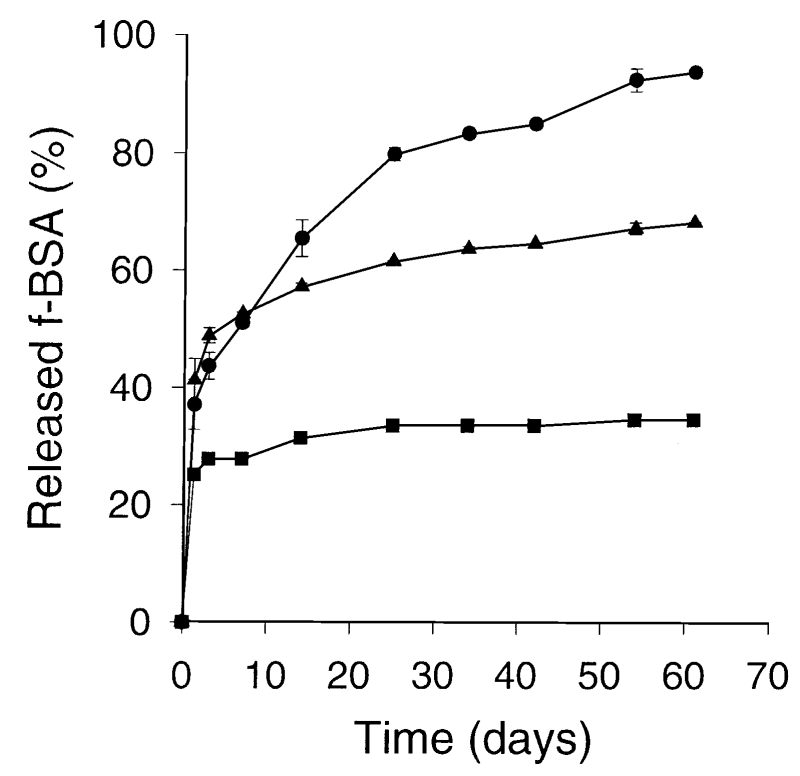

Figure 5. Cumulative release kinetics of f-BSA from f-BSA/PLA (iv = $1.07 \mathrm{dL} / \mathrm{g}$ ) o/o microspheres ( $\square$ ), f-BSA/PLA $\quad(i v=1.07 \quad \mathrm{dL} / \mathrm{g}) / \mathrm{PEG} 35,000$ o/o microspheres ( $\mathbf{\Delta})$, and f-BSA + histidine + trehalose/PLA (iv = $1.07 \mathrm{dL} / \mathrm{g}) /$ PEG35,000 o/o microspheres (O) at $37^{\circ} \mathrm{C}$. (average $\pm \mathrm{SD}, n=3$ ). 
Table 3. Effect of PEG35,000, Histidine and Trehalose on f-BSA Aggregation in Microspheres After 60-day Release in $\mathrm{PBST}$ at $37^{\circ} \mathrm{C}$

\begin{tabular}{|c|c|c|c|c|}
\hline Antigen Formulations & $\begin{array}{c}\text { Total f-BSA } \\
\text { Released }(\%)^{g}\end{array}$ & Soluble f-BSA $(\%)^{d, g}$ & $\begin{array}{l}\text { Non-Covalent and } \\
\text { Disulfide-Bonded } \\
\text { Aggregates }(\%)^{e, g}\end{array}$ & Recovery $(\%)^{g}$ \\
\hline f-BSA/PLA ${ }^{a}$ & $34.5 \pm 0.0$ & $27 \pm 2$ & $5 \pm 2$ & $65 \pm 3^{f}$ \\
\hline $\begin{array}{l}\text { f-BSA/PLA/PEG } \\
\text { blend } d^{a, b}\end{array}$ & $68.1 \pm 0.1$ & $16.4 \pm 0.8$ & $2.7 \pm 0.2$ & $87.2 \pm 0.2^{f}$ \\
\hline $\begin{array}{l}\text { f-BSA+His+Tre/PLA/ } \\
\text { PEG blend }{ }^{a, b, c}\end{array}$ & $93.7 \pm 0.3$ & $17.7 \pm 1.1$ & 0 & $111 \pm 1$ \\
\hline
\end{tabular}

${ }^{a}$ Theoretical protein loading was $6 \%$; PLA (iv $\left.=1.07 \mathrm{dL} / \mathrm{g}\right)$ concentration $(\mathrm{w} / \mathrm{v})$ during encapsulation was $20 \%$ in the absence of PEG and $14 \%$ in the PLA/PEG blend.

${ }^{b}$ PEG (MW 35,000) concentration (w/v) was 6\%.

${ }^{c}$ Histidine and trehalose were co-encapsulated in the microspheres. The histidine:trehalose:f-BSA weight ratio is 1:1:2.

${ }^{d}$ Soluble in PBST.

${ }^{e}$ Soluble in combined denaturing and reducing agents $(6 \mathrm{M} \mathrm{GnCl}$ and $10 \mathrm{mM} \mathrm{DTT})$.

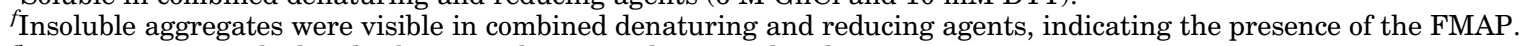

${ }^{g}$ Percentage was calculated relative to the original encapsulated protein amount.

neutral microclimate $\mathrm{pH}$ and high water content was utilized to encapsulate f-BSA by the o/o method.

As seen in Figure 4, after preparation microspheres prepared from the PLA/PEG blend had spherical and smooth surfaces. In Figure 5, the effect of PEG blending and coencapsulation of histidine and trehalose on the release kinetics of $\mathrm{f}-\mathrm{BSA}$ is displayed. In the absence of additives, $34.5 \%$ of f-BSA was released out of the microspheres after 60 days of incubation. The solubility of residual f-BSA remaining in microspheres was analyzed and listed in Table 3. Most remaining fBSA formed insoluble aggregates (27\% soluble in PBST; 38\% formed aggregates). Five percent of fBSA aggregates were identified as soluble in combined denaturing and reducing solvents, indicating most unreleased f-BSA formed formaldehyde-mediated aggregates. After blending PLA with PEG, both release rate and total releasable amount of f-BSA was increased. After 60 days of incubation, formaldehyde-mediated aggregates formed in microspheres were reduced to $<15 \%$. The FMAP was significantly inhibited likely because of the increased water content in microspheres, which is consistent with our previous finding that FMAP is less favorable at high water content. ${ }^{7,8}$ With coencapsulation of histidine and trehalose (the ratio of f-BSA/ histidine/trehalose was 1:0.5:0.5), the FMAP was completely halted. For f-BSA, $93.5 \%$ was continuously released out of polymer device and unreleased f-BSA fraction remained soluble in microspheres.

\section{CONCLUSIONS}

The model formalinized antigen, f-BSA forms aggregates mainly through the formaldehydemediated aggregation pathway when encapsulated in PLGA microspheres. The coencapsulation of histidine and trehalose and the application of o/ o encapsulation technique significantly inhibited the aggregation of f-BSA in the PLGA system. Finally, 2-month controlled release of f-BSA was attained by using the blend of PLA and PEG polymers. Stabilization and controlled release of $\mathrm{f}$ BSA provides a new avenue for the formulation design of formalinized protein antigens, including TT and DT, and other formalinized vaccine antigens in the pipeline for single-dose vaccine development.

\section{NOMENCLATURE}

$\begin{array}{ll}\text { BSA } & \text { bovine serum albumin } \\ \text { DTT } & \begin{array}{l}\text { dithiothreitol } \\ \text { formaldehyde-mediated aggregation } \\ \text { pMathway }\end{array} \\ \text { f-BSA } & \begin{array}{l}\text { formalinized bovine serum albumin } \\ \text { f-Rnase }\end{array} \\ \text { formalinized ribonuclease A } \\ \text { BSA } & \text { bovine serum albumin } \\ \text { TT } & \text { tetanus toxoid } \\ \text { DT } & \text { diphtheria toxoid } \\ \text { PLGA } & \text { poly(lactide-co-glycolide) } \\ \text { PLA } & \text { poly(D,L-lactide) } \\ \text { PEG } & \text { poly(ethylene glycol) } \\ \text { PVA } & \text { poly(vinylalcohol) }\end{array}$




$\begin{array}{ll}\text { PBST } & \begin{array}{l}\text { phosphate buffer saline containing } \\ 0.02 \% \text { Tween } 80^{\circledR}\end{array} \\ \text { GnCl } & \begin{array}{l}\text { guanidine-HCl } \\ \text { water-in-oil-in-water double }\end{array} \\ \text { emulsion-solvent evaporation } & \text { em } \\ \text { o/o } & \begin{array}{l}\text { oil-in-oil single emulsion-solvent } \\ \text { extraction }\end{array} \\ \text { iv } & \text { inherent viscosity }\end{array}$

\section{ACKNOWLEDGMENTS}

This research was supported in part by a grant from the Pharmaceutical Research and Manufactures of American Foundation (PhRMA).

\section{REFERENCES}

1. Schwendeman SP, Costantino HR, Gupta RK, Langer R. 1997. Peptide, protein, and vaccine delivery from implantable polymeric systems. In: Park K, editor. Controlled drug delivery, challenges and strategies. Washington, DC: The American Chemical Society, pp. 229-267.

2. Hanes J, Cleland JL, Langer R. 1997. New advances in microsphere-based single-dose vaccines. Adv Drug Del Rev 28:97-119.

3. Gupta RK, Chang AC, Siber GR. 1998. Biodegradable polymer microspheres as vaccine adjuvants and delivery systems. Dev Biol Stand 92:6378 .

4. O’Hagan DT, Singh M, Gupta RK. 1998. Poly(lactide-co-glycolide) microparticles for the development of single-dose controlled-release vaccines. Adv Drug Del Rev 32:225-246.

5. Alonso MJ, Gupta RK, Min C, Siber GR, Langer R. 1994. Biodegradable microspheres as controlledrelease tetanus toxoid delivery systems. Vaccine 12:299-306.

6. Xing DK, Crane DT, Bolgiano B, Corbel MJ, Jones C, Sesardic D. 1996. Physicochemical and immunological studies on the stability of free and microsphere-encapsulated tetanus toxoid in vitro. Vaccine 14:1205-1213.

7. Schwendeman SP, Costantino HR, Gupta RK, Siber GR, Klibanov AM, Langer R. 1995. Stabilization of tetanus and diphtheria toxoids against moisture-induced aggregation. Proc Natl Acad Sci USA 92:11234-11238.

8. Jiang $\mathrm{W}$ and Schwendeman SP. Formaldehydemediated aggregation of protein antigens: Comparison of untreated and formalinized model antigens. Biotech Bioeng 70:507-517.

9. Aguado MT. 1993. Future approaches to vaccine development: Single-dose vaccines using controlledrelease delivery systems. Vaccine 11:596-597.
10. Johansen P, Men Y, Audran R, Corradin G, Merkle HP, Gander B. 1998. Improving stability and release kinetics of microencapsulated tetanus toxoid by coencapsulation of additives. Pharm Res 15:1103-1110.

11. Tobío M, Nolley J, Guo Y, McIver J, Alonso MJ. 1999. A novel system based on a poloxamer/PLGA blend as a tetanus toxoid delivery vehicle. Pharm Res 16:682-688.

12. Sanchez A, Villamayor B, Guo Y, McIver J, Alonso MJ. 1999. Formulation strategies for the stabilization of tetanus toxoid in poly(lactide-co-glycolide) microspheres. Int J Pharm 185:255-266.

13. Schwendeman SP, Costantino HR, Gupta RK, Tobio M, Chang AC, Alonso MJ, Siber GR, Langer R. 1996. Strategies for stabilising tetanus toxoid towards the development of a single-dose tetanus vaccine. Dev Biol Stand 87:293-306.

14. Greenspan L. 1977. Humidity fixed points of binary saturated aqueous solution. J Res Natl Bureau Stds - A Phys Chem 81A:89-96.

15. Schimd FX. 1990. Spectral methods of characterizing protein conformation and conformational changes. In: Creighton TE, editor. Protein stucture: A practical approach. Oxford: IRL, pp. 145-153.

16. We have previously reported that BSA aggregates in PLGA were completely soluble in denaturing solvent, indicating its noncovalent feature. ${ }^{19}$ Depending on the release conditions, status of the free thiol groups in BSA, etc., we also observe a minor contribution from thiol-disulfide interchange to the aggregation of BSA. This aggregation is uniformly inhibited by increasing the microclimate $\mathrm{pH}$ in the polymer.

17. The development of acidic microclimate $\mathrm{pH}$ in PLGA systems has been recognized to be a common deleterious condition for acid-labile proteins. The extent to which microclimate acidity develops is also known to be strongly dependent on several factors, such as initial monomer content, glycolide content, polymer permeability, and the presence of co-encapsulated buffering species.

18. Zhu G, Schwendeman SP. 2000. Stabilization of proteins encapsulated in cylindrical poly(lactide-coglycolide) implants: Mechanism of stabilization by basic additives. Pharm Res 17:350-356.

19. Zhu G, Mallery SR, Schwendeman SP. 2000. Stabilization of proteins encapsulated in injectable poly(lactide-co-glycolide). Nat Biotechnol 18: 52-57.

20. Paliwal R, London E. 1996. Comparison of the conformation, hydrophobicity, and model membrane interactions of diphtheria toxin to those of formaldehyde-treated toxin(diphtheria toxoid): Formaldehyde stabilization of the native conformation inhibits changes that allow membrane insertion. Biochemistry 35:2374-2379. 
21. Schwendeman SP, Cardamone M, Brandon MR, Klibanov A, Langer R. 1996. Stability of proteins and their delivery from biodegradable polymer microspheres. In: Cohen S, Bernstein H, editors. Microparticulate systems for the delivery of proteins and peptides. New York: Marcel Dekker, Inc., pp. 1-49.

22. Volkin DB, Staubli A, Langer R, Klibanov AM. 1991. Enzyme thermoinactivation in anhydrous organic solvents. Biotech Bioeng 37:843-853.

23. Carrasquillo KG, Cordero RA, Ho S, Franquiz JM, Griebenow K. 1998. Structue-guided encapsulation of bovine serum albumin in poly(-lactic-co-glycolic) acid. Pharm Pharmacol Commun 4:563-571.

24. Costantino HR, Schwendeman SP, Griebenow K, Klibanov AM, Langer R. 1996. The secondary structure and aggregation of lyophilized tetanus toxoid. J Pharm Sci 85:1290-1293.
25. Cleland JL, Powell MF, Shire SJ. 1993. The development of stable protein formulations: a close look at protein aggregation, deamidation, and oxidation. Crit Rev Ther Drug Carrier Syst 10: 303-377.

26. Jiang W, Schwendeman SP. 2001. Stabilization and controlled release of bovine serum albumin encapsulated in poly(D,L-lactide) and poly(ethyl ene-glycol) microsphere blends. Pharm Res 18: $878-885$.

27. It is important to note that because we have recovered no measurable aggregates in the fresh microspheres and very low amounts of aggregates in the early time points in Figure 2, it is highly unlikely that any significant aggregation occurred during our procedure of extracting the protein from the polymer. 